ing than those described. On the other hand, when the patient is a pregnant woman, or one who has just aborted or miscarried, the administration of the treatment is unendurably appalling to sympathetic friends.

By far the most convenient arrangement I have ever used is a tub of ordinary shape and full size, set on a low truck with small wheels, and placed two or three feet from the patient's bed. It is kept sufficiently full of water for the purposes of a complete immersion bath. It is not necessary to change the water oftener than once a day unless it be accidentally polluted; and, standing in the sick-room in ordinary weather it maintains about the proper temperature, 65 or $70 \mathrm{~F}$., for immediate use. If it is warmer than this it can be easily cooled. I shall leave to others the description of details, premising that fidelity to every detail is indispensable as a basis of fair criticism.

In the best hospitals in the world in which this treatment is systematically used, the mortality rate of typhoid fever is 7 or 7.5 per cent.; and, in hospitals of like grade in which the treatment is not employed, the mortality rate of the disease is 14 per cent. To be sure, certain hospitals quote a rate of 1 or 2 per cent., based on a small number of cases or on a large number of mild ones; but a private practitioner who has never employed hydrotherapy at all may be able to do the same thing. It is not to be forgotten that the fatality of typhoid fever is extremely variable in different years and in different localities during the same year. During the past seven or eight years the disease has been uncommonly mild; and I doubt very much if the general mortality rate of private practice during this period has reached even the smallest figure named.

If immersion bathing is refused, or impracticable, sponging with very cold water, ice-water, is a fair substitute. Indeed, in my view, efficient sponging is to be preferred in many cases, when the patient is very weak, or pregnant, or has a complicating pneumonia, and the effects of the immersion bath are overwhelming. In severe cases the sponging with ice-water may have to be continued twenty or thirty minutes and be repeated crery three or four hours. A rectal temperature of 103 F., or above. is, in this country, usually regarded as the critical point requiring the immersion or the sponge bath. Vigorous rubbing is an important feature of both methods of treatment, in which respect they are much superior to the cold pack.

Perfunctory sponging with tepid water, without reference to effects, is of little consequence apart from maintaining an empty show of activity. An important result of the cold bath is the increased activity of elimination as shown by the increased toxicity of the urine following the bath.

3160 Indiana Avenue.

\section{TREATMENT OF MEDICAL COMPLICATIONS OF TYPHOID FEVER.*}

\section{BY GEORGE W. WFBSTER, M.D.}

(IIICAGN.

In the consideration of any phase of the typhoid fever question, we are confronted by, and must take cognizance of, the following facts: 1 , that the disease is a general infection by a germ which circulates freely in the blood and may find lodgment, and cause inflammatory reaction in almost all organs and tissues of the body, and that it possesses pyogenetic properties; 2,

*Read in a Symposium on Typhoid Fever, at a joint meeting of the Chicago Medical Socicty and the Chicago Society of Internal Medicine, Jan. 31, 1900. that typhoid infection predisposes to the occurrence of secondary infection, especially by the pyogenic organisms, as the streptococcus, staphylococcus, and also by the pneumneoccus, the bacterium coli and the bacillus of tuberculosis. Furthermore, there seems to be no uniformity in the use of the term, "complication." Is it to be employed in designating all the unusual manifestations of the affection when due to the typhoid bacillus alone, or only to those complications which follow secondary infection?

I shall, as far as possible, limit my remarks to the medical complications which are now commonly attributed to secondary infection in connection with the circulatory and the respiratory systems. The most important ones in connection with the circulatory organs are endocarditis, pericarditis, myocarditis, arterial and venous thrombosis, embolism, and hemorrhage. While endocarditis and pericarditis are rare, and although they are sometimes caused by the typhoid bacillus, they are more frequently due to secondary infection by the pyogenic organisms. When the result of the blood examination demonstrates this to be the case, or even when it is suspected, the beneficial results of the treatment by means of antistreptococcic serum, together with the fact that it is harmless, would seem to justify a more extended use. It is difficult to formulate general rules for treatment, but we may be safely guided by general principles. In the treatment of acute endocarditis, the most important factor is rest, absolute rest in all that this term implies, physical, mental, sufficient sleep, and reasonable freedom from pain. Such patients should not be permitted to make the slightest exertion, nor should even the head be lifted from the pillow, and no movement or turning or unnecessary disturbance of the patient should be permitted. Food should be given through a glass tube. Even the bedpan may have to be dispensed with and a cloth substituted.

Chilling of the surface must be carefully guarded against, when artificial warmth may be necessary. Occasional hot applications and counter-irritation in the precordial region are of advantage, and Abram?s theory of the heart refiex offers a reasonable explanation of its mode of action. Such patients should be kept in bed for at least thirty days after the complete disappearance of the acute disease of which the endocarditis is a complication or sequel. The neglect of this rule very frequently leads to disaster in cases which might otherwise have recorered with only slight leakage.

In the drug treatment, it is as important to know what not to do as to know what drugs to administer. When the heart's action is becoming enfeebled, there is restlessness, some breathlessness, pain and anxiety. There is often a temptation to give digitalis; it should not be given; it is not only useless, but absolutely pernicious and dangerous. This is true not alone of acute endocarditis, but also of myocarditis, the latter being suspected when there is diffuse, feeble impulse and the first sound is greatly weakened or absent. Even in the ordinary weak heart of typhoid fever, where the heart does not respond to its use within twenty-four hours, it should be discontinued. In such cases, where it is being administered, if the arterial pressure becomes high or the heart irregular, or if the beats become interspersed with small ones, its use should be abandoned.

Opium in some form, either morphin or heroin, is the most valuable drug in the treatment of acute endocarditis. The size of the dose will be determined by the degree of restlessness and pain and the amount of 
sleep. The same rules apply in myocarditis and pericarditis. The next in order of efficiency and value are strychnia, oxygen, ammonia, caffein, camphor hypodermically in sterilized olive-oil. Nitroglycerin is useful in those cases in which the blood-pressure is too high.

The treatment of the chronic valvular lesions, or pericarditis with large effusions, is not within the scope of this paper's discussion.

In the treatment of thrombosis, the most important point is to know what not to do. Whenever it occurs, the patient should be kept as quiet as possible, and movements that are not absolutely necessary should be interdicted, on account of the danger of embolism. All rubbing unguents or liniments, massage and bandaging are especially harmful and dangerous. If in one of the extremities, it should be covered with guttapercha tissue and this surrounded by a layer of cotton wool, the limb elevated and immobilized. We thus meet the indications. Such a patient should be kept quiet in bed for at least six weeks, in order to guard against the danger of embolism. When the venous pressure is high as the result of lowered arterial pressure, careful support of the heart will get the blood out of the venous into the arterial side of the circulation, and thus lessen the danger and promote the comfort and safety of the patient. The same general rules apply in embolism, whether due to endocarditis or thrombosis.

The chief medical complications of the respiratory system are ulceration of the pharynx and larynx, bronchitis, pleurisy with or without effusion, empyema, gangrene, bronchopneumonia and Iobar pneumonia. I will make a brief reference to the last two only. The first general rule is that no matter whether the pneumonia be of the bronchopneumonic type or of the lobar variety, it should be treated essentially as though it were the dominating lesion instead of a complication. The second is that each case must be carefully individualized and treated according to the condition of the patient, and in accordance with certain well-recognized principles. Some cases are mild and require little except careful watching and nursing, with perhaps a little skilful medication. The third point is that, until recently at least, we know nothing of the prophylaxis of the disease and were unacquainted with any curative treatment. It was simply a question of vitality.

The results of Pane in Italy and Antonio Fanoni of New York in the use of Pane's serum lead us to believe that the serum treatment of pneumonia is a distinctly curative measure. Fanoni reports fifteen consecutive cases, all cured. The results of treatment by other varieties of serum in this country are not so encouraging. But aside from this specific medication, we must bear in mind that we are dealing with an acute, short, self-limited disease, in which recovery is a question of vitality, and that as we know of no cure, we are not treating a disease, but a patient with an ailment. This ailment is often severe, frequently fatal in its event, the vitality lowered, resistance taxed to the utmost by pain, want of sleep, extreme toxemia and impoverished blood, high temperature and an overworked, weakened right heart. These furnish the indications for rational treatment. It should be our aim to keep the patient reasonably free from pain, see that he has a sufficient amount of sleep, promote his nutrition and maintain his strengtl, keep the temperature within due bounds and, above all, support the right heart.

For the relief of pain, hot applications may be used when the temperature is not too high. They relieve the pain and reflexly expand the lung. A wet compress is often all that is necessary. Ice massage is a very potent remedy and the relief is usually prompt and decided. I do not favor the continued application of cold. Opiates should be used with extreme care and in the smallest dose that will give relief.

Rest and sleep may be secured by the use of chloral, chloretone, sulfonal, heroin, or morphin in some cases. Restlessness and delirium are sometimes overcome by hyoscin. A water bag filled with ice-water and placed under the back of the neck will lower temperature, soothe the nervous system and promote rest and sleep. Warm sponging, especially in children, has a very soothing, quieting effect.

The diet should be rather more supporting and nutritious than the ordinary milk diet of typhoid fever.

The question of temperature is a vexed one, a debatable land where opposing views are in rather sharp contrast. There is no consensus of skilled opinion in regard to either the necessity for, or the best means of, lowering the temperature. When it arrives at a degree which in itself is a menace to life or threatens the disintegration of blood-corpuscles, or seriously interferes with rest, then some means should be adopted to lower it within reasonable limits. The ice bag is a powerful means, but should not be continuously applied. The wet pack to the affected side may be sufficient, or it may be applied to the abdomen. Sponging with warm water will often suffice and should be tried before we resort to more heroic measures. The cold-water bag to the back of the neck is efficacious and often sufficient. If obliged to resort to drugs, then preference should be given to phenacetin.

Last in order, but first in importance, is the support of the right heart. Because of the double toxemia, the high temperature, inadequate rest, and the added burden imposed by the increased resistance in the lung, the right heart becomes the vulnerable point, the "open door" through which death most frequently enters. Our first care should be to see that no foods nor drugs should be given which cause vomiting. This is particularly true of digitalis. The act of vomiting causes a very severe strain on the heart. As soon as the right heart begins to flag, then we should, as the necessities of the individual case demand, use strychnia, digitalis within the limits already indicated, ammonia, oxygen, camphor hypodermically in sterilized olive-oil, caffein. I wish to go on record as strongly in favor of the use of oxygen in pneumonia when there is cyanosis, and particularly where the heart is weak.

When the lungs are rapidly filling up and heart failure seems imminent, then there is nothing of such effciency and potency as a hypodermic injection of $1 / 4$ grain of mornhin. If the blood-pressure is nigh, and this is not often the case, nitroglycerin should be administered; if too low, belladonna should be given.

70 State Street.

$$
\text { DISCUSSIOX ON TYPHOID FEVER. }
$$

DR. Frank S. Jounson-The hospital treatment of this disease has at all times furnished the data on which the usual treatment at different periods has been based. The large number of cases aggregated in hospitals gives the best opportunity to judge of the degree of virulence of the disease, and furnishes opportunity to apply therapeutic measures and to judge of their respective merits. Even carefully compiled hospital reports on special plans of management may be deceptive unless parallel series of cases under expectant management in the same year and under otherwise similar conditions can be observed for comparison. The disease presents so many complex problems to the therapeutist that he is led to accept some one 
phase as the salient feature and to bend his energies in that direction in combating the disease.

The hospital treatment at the present day may be classed under three general plans: expectant, intestinal antisepsis, and heat reduction. Tone of these alone is fully adequate. The invariable practice is a blending of two or all of them. The expectant plan is a partial acquiescence in the inevitable. No radical measures are taken to influence the course of the specific disease. Attention is directed entirely to the preservation of the strength of the patient by careful feeding, tonics and stimulants, and to guard him, as far as possible, against intercurrent and complicating disease. Under this treatment the affection probably runs a nearly natural course. In practice, however, purely expectant treatment is seldom followed: measures belonging to more radical plans of treatment have always seemed indicated in occasional cases. The author presented an elaborate array of statisties and the results under different methods of treatment. The data showed a very wide range of results obtained under the expectant plan. The differences were attributed chiefly to the variations in the severity of epidemics, and to the quality of nursing.

The plan of treatment by intestinal antisepsis was the natural deduction following the discovery of the specific cause of the disease. In the selection of an antiseptic several problems present themselves: 1 . The antiseptic should be one which will destroy the specific germ together with the associated pathogenic and saprophytic organisms. 2. One which will be locally harmless to the tissues. 3 . One which will be non-toxie to the patient.

In the attempt to secure an agent which would fulfil the above indications, a new and hitherto unreckoned pathologic factor presented itself. The infection was not confined to the contents of the alimentary tube, but to the intestinal walls; the lymphaties, the blood, and in fact all the fluids and tissues of the body were infected to a greater or less degree. Still another desideratum was sought for, but so far unsuccessfully, namely, an agent which after absorption and solution in the juices of the body might still have a germicidal or, at least, an inhibitory influence on the typhoid germs and others accidentally present. Intestinal antisepsis only imperfectly limits the quantity of infection. It may be serviceable in checking saprophytic growths in the alimentary canal and prerenting complicating intoxications. The plan of treatment has berin almost invariably modified by measures to promote the evacuation of infectious and toxic material from the bowels. Statistics of treatment by intestinal antisepsis were given.

Hydrotherapy is perhaps most efficiently carried out by means of the bed or the tub bath. In the usual hospital practice tepid, cool or cold spongings with friction, or the tepid, cool or ice-cold pack is relied on in preference to the bath in all excepting the most urgent cases.

The objects primarily sought by the external application of cold are reduction of body temperature; stimulation of the nervous system; stimulation of respiration; stimulation of circulation. The secondary objects are increase of the exchange of gases, which is estimated to be proportionate to the reduction of temperature; increase in the oxidation of harmful products of tissue destruction and their conversion into less harmful exerementitious matters.

The author gave the results of the hospital treatment by hydrotherapy, as collected from various hospitals both in this country and in Europe.

A few instances of unusual management of the disease are on record in the current literature. Paget reports a series of one hundred cases at Freemantle Hospital in 1897 , with no deaths. The treatment consisted in the use of salad oil in large quantitics, to which was ascribed a protective effect on the mucous membrane of the bowel.

It is common practice in the majority of institutions to administer alcohol in delirium; when the tongue is brown and dry: when the heart is feeble, and when the pulse is irregular or intermittent. At Maidstone, England, in the epidemic of $189-1985$ cases were treated, with a mortality of 7.5 per cent. A milk diet was relied on. Boric acid was administered, and whisky is said to have been given as a routine measure in quantities varying from 16 to 20 ounces per diem.

With reference to diet in typhoid fever, it is the almost uni- versal practice to administer only liquid or very soft food. In hospital typhoid-fever dietaries milk is the chief article and is supplemented by meat broths and juices, farinaceous gruels, soft egrss, etc. Articles of diet must occasionally be selected to meet the individual digestive capabilities. The apparent wisdom of adhering to the liquid diet rule is forced on practitioners from time to time by the result of dietetic indiscretion during the height of the fever and in convalescence. A few practitioners have undertaken to disprove the need of the rigid precautions usually observed as regards diet. From the data given by the author of this paper, it would appear that many facts have been firmly established respecting the management of typhoid fever. There is still a broad field for investigation, and many details are as yet under judgment. Confusion in results arises largely from the protean aspects of the disease.

Dr. A. H. BURR-In the selection of any therapeutic measure for the relief or cure of any disease, two paramount questions are to be solved: 1 . What are the essential indications for treatment in a given disease? 2. What are the physiologic properties of the remedy chosen to meet these indications?

This is the essence of rational medicine. In the absence of any known specific treatment for typhoid fever, these two card. inal propositions must be carefully considered and skilfully co-ordinated, otherwise we must grope about under empiricism. Unfortunately for us, the clearing up of the etiology of typhoid fever has not solved the problem of how to cure it. What, then, are the essential indications in typhoid fever calling for treatment? Virchow, nearly fifty years ago, was the first to declare the fundamental doctrine that fever is a condition of vasomotor paresis. This is pre-eminently so in typhoid intoxication. The improved organic functions; the retarded metabolism; the defective elimination are chiefly the resultants of vasomotor paralysis, or lowered cardiac and vascular tension. Manifestly then the indications call for remedies that can safely and efficiently stimulate the vasomotor system, and support it in its task of tiding the patient through to the establishment of the self-limiting inmunity.

What are the physiologic properties of the Brand kath, which make it the quickest, safest and most efficient vasomotor stimulant known? The physiologic properties of the cool bath have been as carefully worked out, and as definitely established by Winternitz of Vienna, the father of scientific hydrotherapy, and verified by other scientists, as have been the physiologic properties of strychnia or belladonna.

T'o get a clear knowledge of these, three laws of hydriatics must be kept in mind: 1. The brief application of hot or cold water is stimulative. 2. The prolonged application of hot or cold water is sedative. 3. The degree of stimulation or sedation is directly as the temperature varies above or below that of the body.

Thse are its thermic properties, also the physiology of the cutaneous surface on which the cool water is to act. Let us remember that the skin is the greatest sensory organ of the body. The impact of cold water on the cutaneous nerves in stantly sets up a strong reflex stimulation of the respiratory centers. Deep spasmodic inhalations take place at once, which soon settle down to stronger rhythmic action. This at once increases elimination of carbon dioxid and absorption of oxygen.

Let us again remember that the skin is the greatest vesicular organ of the body; that it is capable of containing in its vessels one-third of the entire blood of the body. The impact of cool water briefly applied and repeated over and over again in its effects by the rubbing, instantly stimulates muscular contraction of the capillaries and arterioles, and the reflex speedily causes the heart to respond in a slower, stronger impulse against improved vascular tension. With an improved circulatory pressure of an improved blood, glandular activities are quickened, elimination of toxins is increased many fold, metabolism improved, and naturally temperature, which was only an expression of toxicity, is correspondingly reduced. Where this plan has been carried out faithfully, noted hospital clinicians the world over are a unit in reporting a reduction from a mortality of 18 to 20 under all other plans to a death-rate of only 7 to 8 per cent.

The failure of the profession at large to adopt the cold water treatment as a general routine plan is largely due to a failure to clearly grasp the two primal questions we have already stated, namely, the essential indications for treatment in 
typhoid ferer, and the physiologic properties of cool water properly applied to meet these indications. For this state of affairs many of our text-books and teachers of medicine are largely responsible, and to my mind much in error.

Permit me to yuote from a few standard works. Dreschfield, who edits the chapter on "Typhoid Fever" in "Allbutt's System of Medicine," says: "Abstraction of heat without mueh diminution of the production of heat is the safest and best way of reducing the temperature, and the application of cold, especially in the form of the cold bath, best fulfills the requirements." Two errors are manifest: 1 , in regard to temperature per se as the important indication to be treated, and 2 , in overlooking the physiologic properties of water as a stimulant in the Brand method, and attributing its benefits to its physical property of abstracting heat. In Wood and Fitz's "Practice," this statement is made. "The most important part of the treatment of typhoid fever is that which has to do with the reduction of the temperature."

When shall we have done with this hoary-headed error of combating a temperature symptom instead of the underlying toxemia. It is not pyrexia that ails our typhoid patient, but intoxication, from a general infection. Osler throws a glimmer of light on the subject in his text-book, when he says: "The beneficial action is not so much special and antipyretic as general tonic and roborant." Nowhere do these authors give us the rationale of the cold bath, although esteeming its benefits most highly. Farly resort to the bath, to my mind, is just as important in securing the lowest possible death-rate in typhoid fever as the early administration of antitoxin in diph theria. Temperature alone should not be our guide, but con stitutional conditions.

Dr. Fenton B. Turck referyed to congestion of the splanch nic vessels, particularly of the mesenterics, and anemia of the extremities, accompanied by more or less asphyxia, the nails of the patients assuming a bluish color due to venous congestion. Large quantities of water in the colon in a patient in whon there is splanchnic congestion with more or less cardiac disturbance are contraindicated. The expulsion of large quantitie of water from the bowel produces fatigue and increases atony of the bowel. Dr. Turek then alluded to several experiments which he had carried on to determine the beneficial effects of the alternate use of hot and cold water in the treatment of cases of typhoid fever. The use of a small quantity of cold watersay 300 c.c.-.-.for five minutes does not orer-distend the bowels at any time, in his opinion, and immediate stimulation is effected, with slight dilatation of the arterioles. There is like wise stimulation of the sweat-glands, increase of blood-pressure, and equalization of the circulation.

Dr. I. N. Love, St. Jouis, said that the rules which physicians apply in hospital practice can not be applied in private practice. In every case of typhoid fever the physician recognizes the importanee of individualization and of treating the victim more than the disease, recognizing the fact that each individual is a law unto himself whether he has typhoid fever or appendieitis. He has unlearned a great deal about this disease, and said that he fully realizes it is a question of good managernent rather than medication. If he were compelled to limit himself to any one thing in the treatment of this affection, he would use water internally and externally. There are management of cases of typhoid fever, and these were elimination, intestinal drainage, nutrition, and tranquilization. Each point he discussed at some length.

Dr. George W. MoCasker, Fort Wayne, Ind., said that elim ination is by far the most important thing in the treatment of typhoid ferer. and any method which improves and increases four points of the compass which need to be kept in mind in the elimination tells for the benefit of the patient. Per contra, anything like the coal-tar derivatives, which tell against patients, should be damned to perdition in his opinion. He does not think they are justified in the treatment of this disease. Physicians have to face the problem of getting rid of the toxins; they ean not prevent their formation, and such meas. ures as will eliminate the toxic products from the body should be employed with vigor. Patients should be adequately nourished. The results from the administration of semisolid food appear to have been so good in many instances that physicians should feel eneouraged to feed their typhoid fever patients nuch more in the future than they have in the past.

DR. Jour A. Rosisox called attention to a point which he has noticed in the treatment of typhoid fever during the stage of convalescence, and which tends to strengthen the theory of the good effects of solid food. In a number of cases of typhoid fever, after all the acute symptoms had disappeared, and yet patients had a slight rise of temperature every evening, say from 100 to $101 \mathrm{~F}$., and were extremely anxious to partake of food or were hungry, by giving them solid food the temperature immediately dropped to normal. This he explains on the ground that the disease had practically terminated, and that the rise of temperature each day was due to hunger.

\section{CRANIECTOMY FOR EPILEPTIFORM SEIZ- URES AND GRAVE MEN'TAL DISTURB- ANCES FOLLOWING HEAD INJURY WITHOLT APPARENT FRAC- TURE. WITH REPORT OF \\ A CASE.* \\ BY H. REINEKING, M.D. \\ SURGEOX .C. \& N.-W. R'Y. \\ SHEBOY(IAX, WIS.}

The following case is reported, partly because it is somewhat unique in the pronounced character of its symptoms, the absence of demonstrable organic lesion, and the promptness and completeness of relief following operative treatment; partly because it illustrates, better perhaps than any description I could give, a rare but important group of morbid phenomena following injury to the cranium; but chiefly for the purpose and with the desire of calling forth an expression of the views and experience of the Fellows of the Academy in regard to this interesting class of head injuries.

C. M., aged 20 years, American, wood carver, single, gave a family history negative as regards any neurotic or other hereditary tendencies. He was well-nourished, not addicted to the use of tobacco or spirituous beverages, had never been sick, never showed any tendency to nervousness, had had no occasion for worry, no overwork, no mental excitement, and up to the time of injury was of a lively and contented disposition. His sexual organs showed nothing abnormal, and he denied a history of masturbation or of seminal losses.

On Mัonday, March 6, 1899, in jumping from a slowly-moving passenger train, he was thrown violently to the ground and partly stunned. According to his own statement, he braced himself on his hands and in raising his head, was struck over the left parietal bone by the sharp corner of an oil-box. He soon recovered from the immediate effects of the fall and blow and went at once to his work. A small scalp wound over the left parietal region healed rapidly and without surgical attendance. For the next twelve days he continued at work, but found himself troubled with headache, soon associated with dizziness. Work became very fatiguing and was no longer enjoyed. His appetite failed and loss of weight was noticeable. Mentally he became somewhat dejected and irritable. These symptoms became gradually more pronounced, so that on the twelfth day after the injury he was obliged to quit his work.

On the following day, without premonitory symptoms other than those just mentioned, he was suddenly seized with violent muscular spasms, affecting, so far as could be learned, the entire system, and resembling an epileptic convulsion. He was thrown to the ground, re-

* Read before the American Academy of Railway Surgeons, Omaha, Neb. Oct. 12-12, 1899 